\title{
Die Bildung des ersten deutschen Nationalstaats und der Übergang zum modernen Wirtschaftswachstum
}

Die Gründung des deutschen Kaiserreichs im Jahre 1871 stellte einen zentralen Schritt in der Bildung des ersten deutschen Nationalstaats dar, die sich über einen längeren Zeitraum von den 1860er bis zu den 1880er Jahren erstreckte. Wie die Bundesrepublik besaß das Kaiserreich einen föderalen Staatsaufbau, aber sein politisches Regime war nicht demokratisch, genauer: Es war teil-parlamentarisch und teil-konstitutionell. Zwar galt sowohl im Reich als auch in vielen Mitgliedstaaten (jedoch nicht in Preußen!) das allgemeine Männerwahlrecht, aber der Reichskanzler war dem Kaiser - und nicht dem Reichstag - gegenüber verantwortlich. Das Staatsoberhaupt konnte für seine Amtsführung nicht zur Verantwortung gezogen werden, es stand somit außerhalb der Verfassung (hier und im Folgenden Burhop, 2011 sowie Pfister et al., 2021).

\section{Merkmale des modernen Wirtschaftswachstums}

In auffälliger zeitlicher Parallele zur Nationalbildung entstanden in Deutschland etwa um 1880 wichtige Merkmale des modernen Wirtschaftswachstums, die in vielerlei Hinsicht bis in die Gegenwart prägend geblieben sind. Zwar ist schon seit den späten 1810er Jahren ein stetiges und sich mit der ersten Phase der modernen Industrialisierung (ca. 1840er bis frühe 1870er Jahre) beschleunigendes Wirtschaftswachstum zu verzeichnen. Angesichts der starken Zunahme der Bevölkerung reichte es jedoch nicht aus, um auch die materiellen Lebensverhältnisse zu verbessern. So waren die Reallöhne um 1880 nicht höher als um 1820.

1880 bis 1913 belief sich dagegen das jährliche Wachstum des realen Bruttoinlandsprodukts pro Kopf auf etwa 1,5\% und somit am oberen Rand der seit 1990 zu beobachtenden Werte. Der stetige Anstieg der Reallöhne deutet zudem darauf hin, dass die Früchte des Wirtschaftswachstums nun auch bei der breiten Bevölkerung ankamen. Weiterhin verlagerte sich das Schwergewicht innerhalb des Industriesektors: Die erste Phase der modernen Industrialisierung war bis in die frühen 1870er Jahre durch eine Dominanz der Infrastrukturentwicklung geprägt, vor allem durch den Eisenbahnbau, der ein starkes Wachstum der Montanindustrie nach sich zog. In der zweiten Phase der modernen Industrialisierung gewannen im späten 19. und frühen 20. Jahr-

(C) Der/die Autor:in(nen) 2021. Open Access: Dieser Artikel wird unter der Creative Commons Namensnennung 4.0 International Lizenz veröffentlicht (creativecommons.org/licenses/by/4.0/deed.de).

Open Access wird durch die ZBW - Leibniz-Informationszentrum Wirtschaft gefördert. hundert der Maschinenbau, die elektrotechnische und die chemische Industrie an Gewicht. Seit den 1890er Jahren nahmen diese drei Branchen auch auf den Weltmärkten eine führende Stellung ein. Das heutige Muster der Einbindung Deutschlands ins globale Wirtschaftsgeschehen datiert somit aus dieser Zeit. Diese neuen Branchen waren kapitalintensiv, aber auch wissens- und fähigkeitsintensiv; seit etwa 1880 war deshalb die Akkumulation von Sachkapital und Humankapital (in Gestalt von Bildung und Forschung) ein wichtiger Treiber des Wirtschaftswachstums.

Wie beförderte die Bildung des ersten Nationalstaats den Übergang Deutschlands ins moderne Wirtschaftswachstum? Die Antworten auf diese Frage kreisen um drei Stichworte: Marktintegration, Regulierung und Föderalismus.

\section{Institutionelle Marktintegration}

Die Reichsgründung brachte die institutionelle Marktintegration, die spätestens seit der Einrichtung des Deutschen Zollvereins (1834) im Gange war, zu einem vorläufigen Abschluss. Ein einheitliches Bürgerrecht und die 1869 erlassene Gewerbeordnung des 1866 gegründeten Norddeutschen Bundes, die anschließend vom Reich übernommen wurde, bewirkten die Niederlassungsfreiheit und die Gewerbefreiheit im gesamten Staatsgebiet. Sie schufen damit einen nationalen Arbeitsmarkt, begünstigten die Kapitalmobilität zwischen Einzelstaaten und eröffneten Unternehmen die Möglichkeit, unter demselben Wirtschaftsrecht in mehreren Mitgliedstaaten Betriebe zu unterhalten. Der Übergang zu einheitlichen metrischen Gewichten und Maßen (1854, 1868 und 1872) erleichterte die Kommunikation über Gütermengen und damit sowohl den Außen- als auch den Binnenhandel. 1866 bis 1871 brachte die zentrale Organisation des Postwesens in der Reichspost eine deutliche Rationalisierung des Nachrichtenverkehrs mit sich. Die Einführung der Mark 1871, die auf einem Goldstandard fußte, und die Gründung der Reichbank (1875) vereinheitlichten die Währung und schufen die Grundlage für eine moderne Geldpolitik.

Prof. Dr. Ulrich Pfister lehrt Sozial- und Wirtschaftsgeschichte der Neueren und Neuesten Zeit an der Westfälischen Wilhelms-Universität Münster. 
Die Relevanz der im Kontext der Reichsgründung erfolgten institutionellen Marktintegration für den Übergang zum modernen Wirtschaftswachstum war allerdings wahrscheinlich begrenzt. Wichtige Integrationsschritte, namentlich die Zollunion, mehrere Maßnahmen zur Vereinheitlichung des Geldwesens und die Schaffung eines gemeinsamen Handelsrechts waren bereits durch zwischenstaatliche Kooperation vor allem im Rahmen des Zollvereins erreicht worden.

Umgekehrt waren einige im Zusammenhang mit der Reichsgründung erfolgte Schritte entweder von relativ geringer Bedeutung oder sie entfalteten erst langfristig eine wirtschaftliche Wirkung. Von Bismarck vorangetriebene Bestrebungen zur Zentralisierung der sich teils in privater Hand, teils im Besitz der Mitgliedstaaten befindlichen Bahnverwaltungen scheiterten. Die Vereinheitlichung der damals für die rasche Übermittlung kurzer Nachrichten sehr wichtige Telegrafie und ihr Zusammenschluss mit der Postverwaltung bis 1876 war wirtschaftlich von geringer Bedeutung, da technische und administrative Standards zum einen durch internationale Kooperation gesetzt wurden, zum andern durch die hohe Konzentration der Anbieter von Netzinfrastruktur zustande kamen. Der Aufbau der Reichsbank schuf zwar dadurch, dass dieses Institut als einziges im ganzen Staatsgebiet mit Filialen präsent war, eine wichtige Grundlage für einen einheitlichen Geldmarkt; kurzfristige Zinssätze glichen sich aber erst in den 1890er Jahren zwischen verschiedenen Gebieten Deutschlands allmählich an. Schließlich ist zu betonen, dass Marktintegration nicht nur durch institutionelle und organisatorische Vereinheitlichung vorangetrieben wurde, sondern auch durch die Entwicklung der Transport- und Kommunikationsinfrastruktur. Der Aufbau dieser Netze war in den frühen 1870er Jahren bereits weit vorangeschritten. Marktintegration durch Infrastrukturentwicklung und institutionelle Vereinheitlichung war deshalb vor allem von den 1810er bis zu den 1860er Jahren zentraler Treiber des deutschen Wirtschaftswachstums. Zwar schritt Marktintegration in der Ära des Kaiserreichs durchaus noch weiter voran, gegenüber der Akkumulation von Sach- und Humankapitel trat sie jedoch als Wachstumstreiber zurück.

\section{Marktregulierung}

Das Stichwort der Regulierung steht für den im Zuge der Nationalstaatsbildung eingetretenen Wandel des Charakters staatlicher Eingriffe in die Wirtschaft. Im Gegensatz zur Marktregulierung lassen sich koordinative Standards relativ problemlos im Rahmen zwischenstaatlicher Kooperation setzen. Wie beschrieben konnte deshalb schon vor 1870 eine Koordination der Handelspolitik - im Sinne der Schaffung einer Zollunion -, der Währungspolitik und des Handelsrechts erfolgen. Tiefergehende regulierende Eingriffe, die oft vergleichsweise komplexe Rechtsmaterien darstellen und deren Vollzug zentrale Behörden erfordern, sind dagegen leichter im Rahmen einer staatlich stärker integrierten Struktur vorzunehmen - im Fall Deutschlands im Rahmen eines Bundesstaats. Neue zentrale, auf bundesstaatlicher Ebene eingerichtete Behörden waren nicht nur die schon erwähnte Reichspost und die Reichsbank, sondern beispielsweise auch das Kaiserliche Patentamt (1877) und das Reichsversicherungsamt (1884). Beide stehen für komplexe Regelwerke, deren Umsetzung im Alltag einheitlich und zentralisiert zu erfolgen hatte.

Die obligatorische Sozialversicherung für Arbeiter - konkret die Krankenversicherung (1883), die Unfallversicherung (1884) und die Alters- und Invaliditätsversicherung (1889) stellt die wichtigste, international weithin beachtete Sozialinnovation der Bismarck-Ära dar. Mindestens in dreierlei Hinsicht trug sie dazu bei, dass die Früchte wirtschaftlicher Entwicklung in den drei Jahrzehnten vor dem Ersten Weltkrieg breiteren Teilen der Bevölkerung zugute kamen. Erstens führte zwar die Einführung der Unfallversicherung dazu, dass Bestrebungen von Unternehmen im Bereich der Unfallverhütung nachließen und Betriebsunfälle zunahmen. Da das ganze jeweilige Versicherungskollektiv für den Schadenausgleich aufkam, bestand für das einzelne Unternehmen kein Anreiz zur Unfallverhütung. Diese nicht zuletzt vom Reichsversicherungsamt beobachtete Fehlsteuerung wurde 1900 durch eine Tarifdifferenzierung behoben, die es einzelnen Unternehmen ermöglichte, den eigenen Versicherungsbeitrag durch erfolgreiche Unfallprävention zu verringern. In der Folge waren auch Betriebsunfälle rückläufig. Zweitens trug die Einführung der Krankenversicherung zur Verlängerung der Lebenserwartung bei, und zwar direkt durch eine Erleichterung des Zugangs zu medizinischen Dienstleistungen breiter Bevölkerungsschichten sowie indirekt durch die Aufklärungsbemühungen von medizinischem Personal und von Krankenkassen, die auf Seiten der Versicherten verbeugendes Verhalten im Alltag stärkten. Drittens verbesserte die obligatorische Alters- und Invaliditätssicherung durch Zwangssparen die Einkommenssituation der älteren Bevölkerungsteile. Sie linderte Altersarmut und ermöglichte es, früher aus dem Erwerbsleben auszuscheiden. Die Entstehung einer dritten Lebensphase hat somit einen maßgeblichen Ursprung in der Einführung der obligatorischen Sozialversicherung.

Die Gründung des Patentamts steht im Zusammenhang mit dem Erlass des Reichspatentgesetzes (1877). Seine Ausarbeitung dauerte nicht zuletzt deshalb lange, weil es die sehr unterschiedlichen älteren Ansätze der Einzelstaaten zum Schutz geistigen Eigentums in einen kohärenten neuen Rahmen zu überführen galt. Es schuf einen nationalen Markt für technologische Innovationen und ermöglichte damit ausländischen Erfindern einen kostengünstigen Zugang zum deutschen Markt. Damit erleichterte es die internationale Verbreitung technologischer Innovationen. Ein weiterer Effekt tritt 
hinzu: Auf Begehren aus dem Kreis der jungen chemischen Industrie wurde eine Bestimmung aufgenommen, laut der in der Chemie keine Produkte, sondern nur Herstellungsverfahren patentiert werden konnten. Dies schuf besonders in der Farbenindustrie einen intensiven Wettbewerb, denn es galt für eine bestimmte Farbe ein möglichst kostengünstiges Syntheseverfahren zu entwickeln. Der regulative Rahmen leistete deshalb der Entstehung der Industrieforschung in der deutschen chemischen Industrie Vorschub und begünstigte deren Aufstieg zur Weltführerschaft. Allgemein stellte die Ausgestaltung des Patentrechts somit einen Faktor dar, der die Entwicklung wissens- und fähigkeitsintensiver Branchen im späten 19. Jahrhundert begünstigte.

Analoges gilt für den Zusammenhang zwischen Kapitalmarktregulierung und der raschen Kapitalakkumulation in den drei Jahrzehnten ab etwa 1880. Die gesetzliche Regelung der Aktiengesellschaft wurde seit der Mitte des 19. Jahrhunderts intensiv diskutiert, aber der Zielkonflikt zwischen Aktionärsschutz und möglichst leichter Kapitalmobilisierung erschwerte die Politikformulierung. Die meisten deutschen Staaten praktizierten ein Konzessionsregime, bei dem die Gründung einer Aktiengesellschaft einer behördlichen Genehmigung bedurfte. Die Reichsgründung schuf ein Gelegenheitsfenster für den Übergang zu einem liberal ausgestalteten Normativsystem. Das vom Norddeutschen Bund 1870 erlassene Gesetz zur Regulierung von Aktiengesellschaften stellte das Ziel der Kapitalmobilisierung in den Vordergrund und sah nur eine schwache Kontrolle des Vorstands durch die Aktionäre vor. Es begünstigte bis 1873 einen Gründungsboom, in dem eine hohe dreistellige Zahl an Aktiengesellschaften neu gegründet wurde. Ihm folgte im Kontext einer weltweiten Wirtschaftskrise ein sogenannter Gründerkrach mit sinkenden Aktienkursen, bei dem die neu gegründeten Aktiengesellschaften ein höheres Risiko als die noch unter einem Konzessionsregime gegründeten Gesellschaften aufwiesen, vom Markt zu verschwinden.

Mit der Krise setzte unter Experten und bei einer interessierten Öffentlichkeit zunächst eine Kritik an den Fehlanreizen des Regimes von 1870, später dann eine breite Reformdebatte ein. Über mehrere Runden und unter Mitwirkung zahlreicher Akteure, unter denen die preußischen Ministerien, der Bundesrat, die Reichsämter, der Reichstag und die Handelskammern besonders der hanseatischen Städte hervorzuheben sind, erfolgte bis 1884 eine Novellierung des Aktienrechts. Sie löste den Zielkonflikt zwischen Aktionärsschutz und Kapitalmobilisierung durch eine Stärkung der Kontrollbefugnisse der Aktionäre. Die damals gewählte Rollenverteilung zwischen Vorstand, Aufsichtsrat und Aktionären hat in den Grundzügen bis heute Bestand. Die getroffene Regelung senkte nachweislich die Transaktionskosten der Überwachung von Vorständen und leistete damit einen Bei- trag zur Entwicklung großer, kapitalintensiv produzierender Unternehmen in den folgenden Jahrzehnten.

Diese Beispiele zeigen, dass mit der Bildung eines Nationalstaats in der Zeit der 1860er bis 1880er Jahre die Fähigkeit der Politik zunahm, komplexe Materien, die tief in die Wirtschaft eingriffen, angemessen zu regeln. Besonders in den Bereichen des Schutzes geistigen Eigentums und des Aktienrechts trug dies zur Entstehung eines modernen Musters des Wirtschaftswachstums bei, das verstärkt auf die rasche Akkumulation von Sachkapital setzte und das durch ein hohes Gewicht von wissens- und fähigkeitsintensiven Branchen gekennzeichnet war.

\section{Föderalismus}

Die dargestellten wirtschafts- und sozialpolitischen Maßnahmen erfolgten auf der Grundlage zunehmend komplexer Vorgänge der Politikformulierung und Entscheidungsfindung. Der oben dargestellte Weg zur Aktienrechtnovelle hat dies kurz veranschaulicht. Das dritte zentrale Stichwort des Föderalismus steht für diesen neuartigen Politikprozess, der über mehrstufige Verfahren unter Einbeziehung zahlreicher auch dezentraler Akteure sachadäquate ökonomische Institutionen hervorbrachte. Das junge Kaiserreich belegt eine These der Institutionenökonomik, wonach ein föderaler Staatsaufbau die Willkür des Zentralstaats begrenzt und dadurch Institutionen von guter Qualität hervorbringt. Diese Wirkung des Föderalismus war wohl nicht zuletzt vor dem Hintergrund des nur teilweise demokratischen Regimes des Kaiserreichs von großer Bedeutung.

Ein funktionierender Föderalismus setzt voraus, dass die Mitgliedstaaten selber öffentliche Güter bereitstellen und auf der Grundlage ihrer regionalen Verankerung auf nationaler Ebene gewichtige, ernstzunehmende Verhandlungsparteien darstellen. Tatsächlich verfügte das Reich über nur wenige Steuereinnahmen - anteilig wenig mehr als der Zollverein -, und die dezentrale Organisation der öffentlichen Finanzen blieb bis in die Anfangszeit der Weimarer Republik erhalten. Mit dem Aufbau eines Nationalstaats gaben Mitgliedstaaten auch keineswegs nur Befugnisse an die zentrale Ebene $\mathrm{ab}$, sondern bauten umgekehrt bestehende Funktionen in ihrem Territorium aus. Es fand somit auf mehreren Ebenen eine Staatsverdichtung statt. Ein wichtiges Beispiel ist das Bildungswesen, das auch damals in die Zuständigkeit der Mitgliedstaaten fiel: Die Gründungsdaten etlicher Technischer Universitäten fallen in die Zeit der 1860er und 1870er Jahre, und im Zuge der Auseinandersetzung mit der Katholischen Kirche im sogenannten Kulturkampf nahmen nach den 1870er Jahren der Staatsanteil bei der Finanzierung der Volksschule sowie die Dichte der staatlichen Schulaufsicht nachhaltig zu. Dies vereinheitlichte und verbesserte die Qualität des Bildungswesens. Zusammen mit dem Patentgesetz 
von 1877 trug die hohe Qualität des deutschen Bildungswesens dazu bei, dass sich Deutschland im späten 19. Jahrhundert zu einem bevorzugten Standort für die Produktion wissens- und fähigkeitsintensiver Produkte entwickelte. Will man aus der Erfahrung des Kaiserreichs Lehren für die heutige Zeit ziehen, so sind die positiven Folgen eines funktionierenden Föderalismus für die Qualität ökonomischer Institutionen und anderer öffentlicher Güter hervorzuheben.

\section{Literatur}

Burhop, C. (2011), Wirtschaftsgeschichte des Kaiserreichs 1871-1918, Vandenhoeck \& Ruprecht.

Pfister, U., J.-O. Hesse, M. Spoerer und N. Wolf (Hrsg.) (2021), Deutschland 1871. Die Nationalstaatsbildung und der Weg in die moderne Wirtschaft, Mohr Siebeck. 